\title{
Histopathological Findings of Hemorrhagic Ganglion Cyst Causing Acute Radicular Pain: A Case Report
}

\author{
Jong-Hyun Park ${ }^{1}$, Soo Bin $\mathrm{Im}^{1}$, Hee Kyung Kim², Sun Chul Hwang ${ }^{1}$, \\ Dong-Seung Shin ${ }^{1}$, Won Han Shin', Bum-Tae Kim ${ }^{1}$ \\ ${ }^{1}$ Departments of Neurosurgery, ${ }^{2}$ Pathology, Soonchunhyang University College of Medicine, Bucheon Hospital, Bucheon, Korea
}

Although juxtafacet cysts of the lumbar spine are being reported with increasing frequency, hemorrhage from a ganglion cyst is rare, and the pathophysiologic mechanism of the hemorrhage from the cyst is still unclear. A 75-year-old male presented with sudden radicular leg pain caused by hemorrhage from the ganglion cyst. Computed tomography revealed bony erosion of vertebral body and multiple punched-out lesions on facets. Magnetic resonance imaging showed the neural structure was compressed by a sharply delineating mass. Capsule and old hematoma with elastic consistency that extended to the epidural space were removed through a paramedian transforaminal approach, which led to the resolution of the patient's symptoms. Histopathologically, chronic inflammation with neovascularization and myxoid degeneration were present in the capsule. Alcian blue staining demonstrated the mixture of mucin and hematoma. The probable pathogenesis of hemorrhage from the cyst was discussed from the unique histopathological findings of surgical specimen.

Key Words: Ganglion cyst $\cdot$ Hemorrhage $\cdot$ Lumbar vertebrae $\cdot$ Mucins $\cdot$ Radiculopathy $\cdot$ Zygapophyseal joint

\section{INTRODUCTION}

Juxtafacet cysts include both synovial and ganglion cysts adjacent to a spinal facet joint ${ }^{10)}$. Although juxtafacet cysts of the lumbar spine are being reported with increasing frequency, hemorrhage from a ganglion cyst is rare and the rate of hemorrhagic incidence is less than $10 \% 0^{1,3,6-9,11,15,16,18-20)}$.

Although the suspected causal factors such as anticoagulation therapy, trauma, and the presence of a vascular anomaly has been proposed, the etiology of the hemorrhage from the juxtafacet cysts is still unclear, ${ }^{6,20)}$. The similar conditions differently called a ligamentum flavum hematoma, juxtafacet cyst hematoma, hemorrhagic synovial cyst and facet apoplexy have been reported ${ }^{11,15)}$. We report a case of hemorrhage into a ganglion cyst causing an acute radiculopathy without known causative factor. The findings of histopathological staining of a surgical specimen were described, which might provide the

- Received: June 28, 2013 - Revised: September 23, 2013

- Accepted: September 26, 2013

Corresponding Author: Soo Bin Im, MD, PhD

Department of Neurosurgery, Soonchunhyang University Bucheon Hospital

1174 Jung-1-dong, Wonmi-gu, Bucheon-si, Gyunggi-do 424-767, Korea

Tel: +82-32-621-5290, Fax: +82-32-621-5016

E-mail: isbrzw@gmail.com

$\otimes$ This is an Open Access article distributed under the terms of the Creative Commons Attribution Non-Commercial License (http://creativecommons.org/ licenses/by-nc/3.0/) which permits unrestricted non-commercial use, distribution, and reproduction in any medium, provided the original work is properly cited. clue for the pathogenesis of the hemorrhage from the juxtafacet cyst.

\section{CASE REPORT}

A 75 -year-old male presented with radicular pain in his left leg. The disabling radicular pain started 2 weeks earlier with no prior trauma or other known causal factors. He had been taking metformin for diabetes mellitus, but no anticoagulants. His medical history included an endoscopic mucosal resection for early gastric cancer 6 years before presentation. Lower back pain also preceded his current symptoms and had been treated conservatively with intravenous ketamine and midazolam.

On physical examination, motor strength was 4 out of 5 for left knee extension and foot dorsiflexion, with no measurable atrophy. The patient had severe radiating pain and hyperesthesia in the $4^{\text {th }}$ and $5^{\text {th }}$ lumbar dermatomes. His straight leg raising test and deep tendon reflex were negative. Coagulation parameters were within the normal range. Plain radiographs of the lumbar spine revealed spondylotic changes, but no instability. Computed tomography (CT) showed bony erosion and a punched-out lesion at the left $5^{\text {th }}$ lumbar facet joint and an extradural mass. Magnetic resonance imaging (MRI) showed a sharp-margined mass with mixed signal intensity eroding the adjacent vertebral body. The mass severely com- 

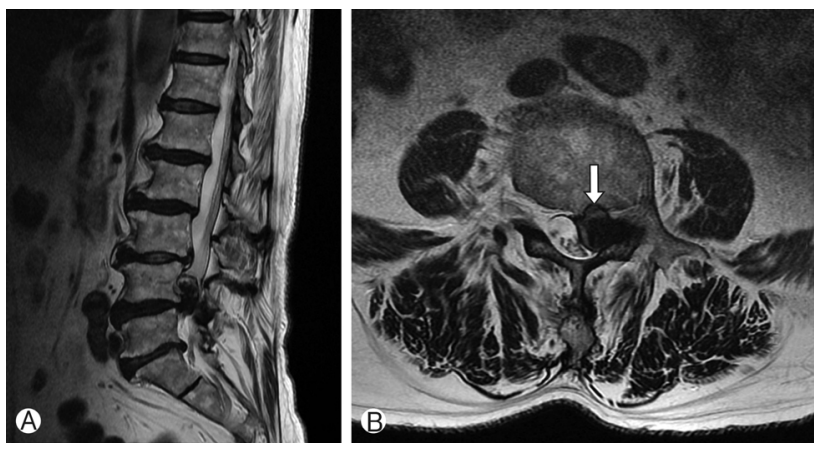

Fig. 1. Preoperative magnetic resonance imaging. (A) Sagittal T2-weighted MRI shows an extradural mass compressing the thecal sac and adjacent nerve root. (B) T2- and T1-weighted MRI shows a well-delineated capsule containing a mixed-signal-intensity lesion that is eroding the adjacent vertebral body (Arrow).

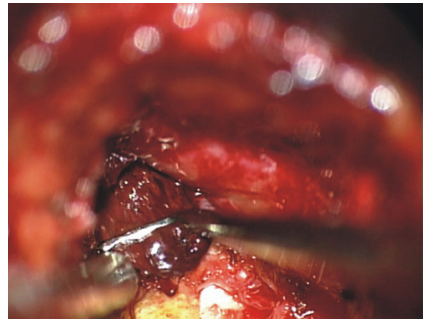

Fig. 2. The articular surface of the facet joint was discolored with dark brown color and extensive hematoma was found between the two layers of ligamentum flavum.

pressed the thecal sac and root (Fig. 1). The patient underwent surgery in the prone position. The lesion was accessed via a paramedian intermuscular approach, and a transforaminal corridor was formed with a facetectomy of the $4^{\text {th }}$ inferior lumbar facet and medial part of the $5^{\text {th }}$ superior lumbar facet. The articular surface of the facet joint was discolored by a hematoma with a dark brown color. We identified an extensive hematoma between the two layers of ligamentum flavum which extended to the epidural space (Fig. 2). The old form of hematoma has more elasticity than usual spinal epidural hematoma. The tough capsule containing the hematoma was connected with the facet joint between the fourth and fifth lumbar vertebra. After removing a large amount of the hematoma and capsule, we could identify the adjacent root and thecal sac. The entire procedure was performed while preserving the spinoligamentous structure at the midline. To prevent instability subsequent to the facetectomy, a pedicle screw was fixed through the intermuscular windows. The percutaneous screw was inserted to the contralateral side. Microscopic examination of the surgical specimen comprising a hematoma and cyst membrane revealed an old cystic lesion with signs of subacute bleeding. Fibrocollagenous tissue with widespread fresh blood and hemosiderin deposits were observed. Chronic inflammation with neovascularization and myxoid degeneration were also present (Fig. 3). Alcian blue stain demonstrated the presence of mucin within the hematoma (Fig. 4). However, there

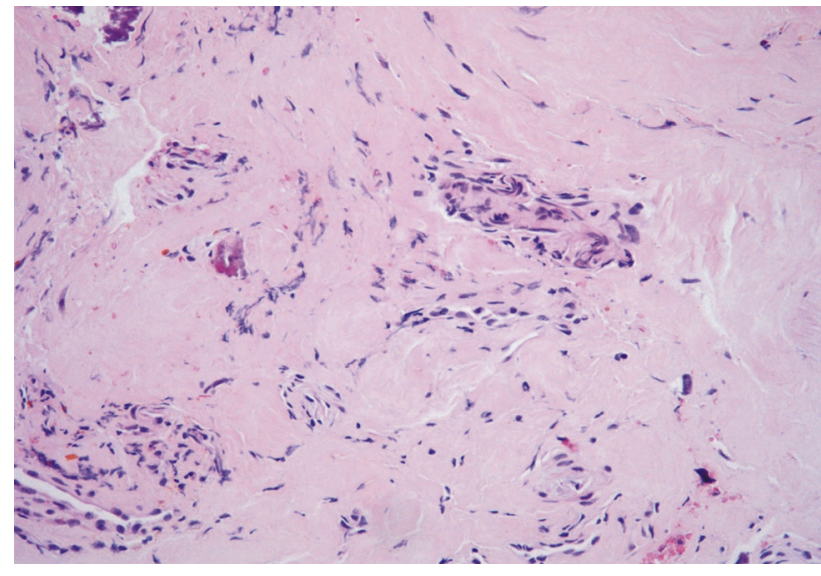

Fig. 3. Histologically, the hemorrhagic ganglion cyst is composed of fibrocollagenous tissue containing widespread multi-stage hemorrhage and hemosiderin deposits. The capsule has no synovial lining. Microscopic image of the cyst wall showed fibrous tissue with myxoid degeneration and an increased number of blood vessels. Microcalcification and macrophages are also present $(H \& E, \times 200)$.

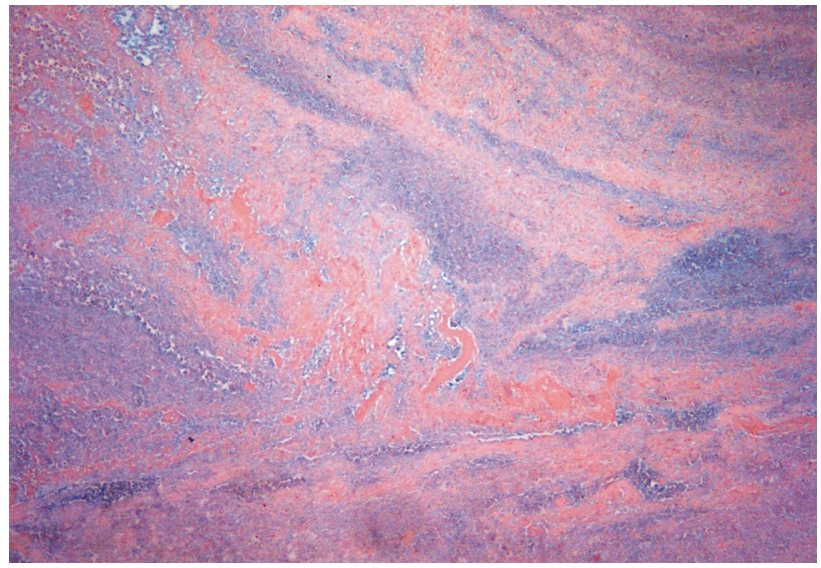

Fig. 4. Microscopic image of the cyst contents showing mixture of mucin and hematoma. The mucin was stained blue with Alcian blue $(\times 200)$.

was no evidence of a synovial lining. The final diagnosis was a hemorrhagic ganglion cyst. Postoperatively, the patient's symptoms and neurological function improved markedly. Follow-up plain radiographs showed no iatrogenic instability or collapse. MRI showed shrinkage of the hematoma and decompression of the dural sac (Fig. 5). During the 2 years of follow-up period, patient had no additional symptom or recurrence.

\section{DISCUSSION}

Recently, lumbar juxtafacet cysts have been reported with 


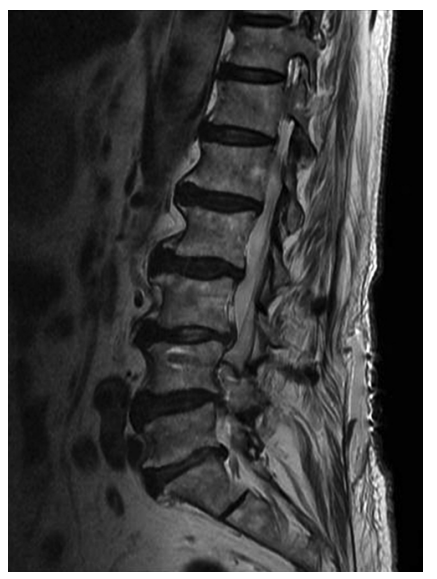

Fig. 5. Postoperative magnetic resonance imaging showing removal of the hematoma and decompression of the dural sac and roots. The metal artifact results from pedicle screw fixation to augment the facetectomy.

increasing frequency because of refinements in neurodiagnostic imaging ${ }^{19)}$. The most common symptom of a juxtafacet hemorrhage is sudden worsening of lower back pain and radicular pain, which occurs most often in the $4^{\text {th }}-5^{\text {th }}$ decade of life. As the symptom of intracystic hemorrhage is similar to ruptured lumbar disc or other degenerative spinal diseases, the diagnosis could be misguided ${ }^{1,12}$. Especially in our case, the discrepancy between the bony erosion, which implied a chronic lesion, and the acute symptom onset made the diagnosis somewhat difficult.

The pathophysiological mechanism of the hemorrhage is still unclear, although the suspected causal factors include anticoagulation therapy, trauma, and vascular anomaly ${ }^{6,20)}$. Trauma can cause the bleeding by the breakage of fragile vessels from the wall of a pre-existing cyst ${ }^{6}$. Evident trauma is reported in only $24 \%$ of cases of the hemorrhage ${ }^{20)}$. This case was not linked to any known causative factors and we favor microtrauma associated with daily activity as the cause of the hemorrhage.

Pathologically, synovial and ganglion cysts are distinguished only by the presence of a synovial lining in the former; clinically, both entities have identical symptoms and prognoses ${ }^{10)}$. Our patient's cyst was categorized as a ganglion cyst due to the absence of a synovial lining. We detected mucin was mixed within the hematoma, which explains the extraordinarily elastic consistency of the hematoma. The cyst wall of hematoma contained neovascularization and macrophages. MRI showed bony erosion of the vertebral body adjacent to the cyst that implies the cyst is chronic lesion and has been expanded in a lengthy period of time. These radiological and histopathological findings support that the hemorrhage was originated from the cyst wall which contains fragile new vessels, and the hematoma bursts into a slow growing mucin containing cyst. The hemorrhagic event from these fragile new vessels might provoke rapid expansion of the cyst and causes acute symptoms.
Although the treatment options include CT-guided aspiration and the percutaneous injection of hyaluronidase, the confident treatment is neural decompression by surgical excision of the hemorrhagic cyst ${ }^{15,18)}$. The need for screw instrumentation for juxtafacet cyst surgery is quite controversial. Some surgeons prefer simple removal of cyst and others recommend instrumentation and fusion ${ }^{2,17}$. Many articles report that the juxtafacet cyst has inherent segmental instability, $4,5,13,14,17$. Niggemann et al. reported high rate of vertebral slip and angular movement in the affected segment ${ }^{14)}$. We consent the juxtafacet cyst is resulted from the long-standing facet degeneration and has an underlying segmental instability. Furthermore, in order to remove the cyst, the facet or laminar should be damaged, we think the augmentation procedure is indispensable. We chose a paramedian transforaminal approach rather than a midline approach, which allows a direct access to the pathologic facet and relevant lesion. In addition, this approach has a benefit of screw fixation without creating dead space in the midline.

\section{CONCLUSION}

We report the case of a hemorrhagic ganglion cyst that caused an acute exacerbation of neurological symptoms mimicking disc rupture or other degenerative spinal diseases. Histopathologically, the hematoma and capsule could explain the pathogenesis of the hemorrhage. Surgical decompression and augmentation via a paramedian transforaminal approach has several benefits in terms of access and stabilization compared with a midline approach.

\section{REFERENCES}

1. Arantes M, Silva RS, Romão H, Resende M, Moniz P, Honavar $\mathrm{M}$, et al: Spontaneous hemorrhage in a lumbar ganglion cyst. Spine (Phila Pa 1976) 33:E521-524, 2008

2. Bashir el F, Ajani O: Management of lumbar spine juxtafacet cysts. World Neurosurg 77:141-146, 2012

3. Brown C, Stambough JL: Epidural hematoma secondary to a rupture of a synovial cyst. Spine J 5:446-450, 2005

4. Bydon A, Xu R, Parker SL, McGirt MJ, Bydon M, Gokaslan $\mathrm{ZL}$, et al: Recurrent back and leg pain and cyst reformation after surgical resection of spinal synovial cysts: systematic review of reported postoperative outcomes. Spine J 10:820-826, 2010

5. Choudhri HF, Perling LH: Diagnosis and management of juxtafacet cysts. Neurosurg Focus 20:E1, 2006

6. Cicuendez M, Alen JF, Ramos A, Lobato RD, Lagares A: Spontaneous hemorrhage into a lumbar synovial cyst. Eur Spine J 19 Suppl 2:S190-192, 2010 
7. Eck JC, Triantafyllou SJ: Hemorrhagic lumbar synovial facet cyst secondary to anticoagulation therapy. Spine J 5:451-453, 2005

8. Eyster EF, Scott WR: Lumbar synovial cysts: report of eleven cases. Neurosurgery 24:112-115, 1989

9. Franck JI, King RB, Petro GR, Kanzer MD: A posttraumatic lumbar spinal synovial cyst. Case report. J Neurosurg 66:293296, 1987

10. Freidberg SR, Fellows T, Thomas CB, Mancall AC: Experience with symptomatic spinal epidural cysts. Neurosurgery 34:989993; discussion 993, 1994

11. Fukuda C, Hirofuji E: Juxta-facet hematoma. J Orthop Sci 12: 597-600, 2007

12. Mizutamari M, Sei A, Fujimoto T, Taniwaki T, Mizuta H: L5 radiculopathy caused by a ganglion cyst of the posterior longitudinal ligament in a teenager. Spine J 9:e11-14, 2009

13. Moon HJ, Kim JH, Kwon TH, Chung HS, Park YK: Cervical juxtafacet cyst with myelopathy due to postoperative instability. Case report. Neurol Med Chir (Tokyo) 50:1129-1131, 2010

14. Niggemann P, Kuchta J, Hoeffer J, Grosskurth D, Beyer HK, Delank KS: Juxtafacet cysts of the lumbar spine: a positional
MRI study. Skeletal Radiol 41:313-320, 2012

15. Nishida K, Iguchi T, Kurihara A, Doita M, Kasahara K, Yoshiya S: Symptomatic hematoma of lumbar facet joint: joint apoplexy of the spine? Spine (Phila Pa 1976) 28:E206-208, 2003

16. Pendleton B, Carl B, Pollay M: Spinal extradural benign synovial or ganglion cyst: case report and review of the literature. Neurosurgery 13:322-326, 1983

17. Sivakumar W, Elder JB, Bilsky MH: Cervical juxtafacet cyst after anterior cervical discectomy and fusion. Neurosurg Focus 31: E19, 2011

18. Summers RM, Quint DJ: Case report 712: Hemorrhagic synovial cyst arising from right L2-3 facet joint. Skeletal Radiol 21: 72-75, 1992

19. Tatter SB, Cosgrove GR: Hemorrhage into a lumbar synovial cyst causing an acute cauda equina syndrome. Case report. J Neurosurg 81:449-452, 1994

20. Xu R, Solakoglu C, Maleki Z, McGirt MJ, Gokaslan ZL, Bydon A: Hemorrhagic synovial cyst: the possible role of initial trauma and subsequent microtrauma in its pathogenesis: case report. Neurosurgery 68:E858-865; discussion E865, 2011 